\title{
Parallel dark-soliton pair in a bistable two-dimensional exciton-polariton superfluid
}

\author{
G. Lerario, ${ }^{1,2, *}$ S. V. Koniakhin,,${ }^{3,4}$ A. Maître $\odot,{ }^{1}$ D. Solnyshkov, ${ }^{3,5}$ A. Zilio, ${ }^{6,7}$ Q. Glorieux $\odot,{ }^{1,5}$ G. Malpuech, ${ }^{3}$ E. Giacobino, ${ }^{1}$ \\ S. Pigeon $\odot,{ }^{1}$ and A. Bramati $\odot^{1,5,1}$ \\ ${ }^{1}$ Laboratoire Kastler Brossel, Sorbonne Université, CNRS, ENS-PSL Research University, Collège de France, \\ 4 place Jussieu, 75252 Paris, France \\ ${ }^{2}$ CNR NANOTEC, Istituto di Nanotecnologia, via Monteroni, 73100 Lecce, Italy \\ ${ }^{3}$ Institut Pascal, PHOTON-N2, Université Clermont Auvergne, CNRS, SIGMA Clermont, Institut Pascal, F-63000 Clermont-Ferrand, France \\ ${ }^{4}$ St. Petersburg Academic University, Nanotechnology Research and Education Centre of the Russian Academy of Sciences, \\ 194021 St. Petersburg, Russia \\ ${ }^{5}$ Institut Universitaire de France (IUF), 1 rue Descartes, 75231 Paris, France \\ ${ }^{6}$ Université de Paris, Laboratoire Jacques-Louis Lions (LJLL), F-75013 Paris, France \\ ${ }^{7}$ Sorbonne Université, CNRS, LJLL, F-75005 Paris, France Université Paris-Diderot, Sorbonne Paris-Cité, Laboratoire Jacques-Louis Lions, \\ CNRS, Batiment Sophie Germain, 75205 Paris CEDEX 13, France
}

(Received 19 March 2020; revised 15 November 2020; accepted 17 November 2020; published 11 December 2020)

\begin{abstract}
Collective excitations, such as vortex-antivortex and dark solitons, are among the most fascinating effects of macroscopic quantum states. However, two-dimensional (2D) dark solitons are unstable and collapse into vortices due to snake instabilities. Making use of the optical bistability in exciton-polariton microcavities, we demonstrate that a pair of dark solitons can be formed in the wake of an obstacle in a polariton flow resonantly supported by a homogeneous laser beam. Unlike the purely dissipative case where the solitons are gray and spatially separate, here the two solitons are fully dark, rapidly align at a specific separation distance, and propagate parallel as long as the flow is in the bistable regime. Remarkably, the use of this regime allows us to relax the phase fixing constraints imposed by the resonant pumping and to circumvent the polariton decay. Our work opens very wide possibilities for studying new classes of phase-density defects which can form in driven-dissipative quantum fluids of light.
\end{abstract}

DOI: 10.1103/PhysRevResearch.2.042041

Promoted by the demonstration of Bose-Einstein condensation (BEC) [1,2], collective excitations have been extensively studied in cold atoms and exciton-polariton systems $[3,4]$. During the past two decades, topologically protected vortices [5-13] and dark solitons [6,14-16] have been successfully demonstrated theoretically and experimentally in both systems.

Exciton-polaritons are bosonic quasiparticles resulting from the exciton-photon strong coupling in microcavities [17], which gives them hybrid properties coming from their components. They have a very light mass inherited from the photon component and they interact with each other due to the exciton-exciton interaction. In recent years, these systems became very attractive in the context of out-of-equilibrium condensates and 2D quantum fluid hydrodynamics [18]. Exciton-polaritons can be created by optical excitation. In particular, it is possible to create metastable supersonic flows

\footnotetext{
*giovanni.lerario@lkb.upmc.fr

†alberto.bramati@lkb.upmc.fr
}

Published by the American Physical Society under the terms of the Creative Commons Attribution 4.0 International license. Further distribution of this work must maintain attribution to the author(s) and the published article's title, journal citation, and DOI. because of the very weak thermal relaxation. This unique possibility allowed the hydrodynamic generation of dark solitons using a supersonic polariton wave packet hitting a structural defect in its in-plane propagation $[14,15]$. Both pulsed and continuous-wave $(\mathrm{cw})$ resonant excitation have been used. In pulsed resonant configuration, the polariton flow can propagate freely after the pulse is over, but its lifetime and propagation distance are relatively short. In $\mathrm{cw}$ resonant configuration, the pump should not spatially overlap the regions where solitons are created (otherwise the phase is imposed by the laser and phase defects cannot exist). As a result, similarly to the pulsed case, the flow strongly decays along the propagation.

The formation of oblique dark solitons within the polariton coherent state has been observed in such configuration [15]. The transverse "snake" instability [19,20], which normally destroys the 2D dark solitons by converting them into chains of vortex-antivortex pairs, is suppressed by the supersonic flow [21]. The 2D solitons are effectively 1D in this case, and the spatial direction along the polariton flow (in-plane wave vector of laser light) is mapped to an effective time. In 1D cavities, stable solitons were also studied and their phase relation with respect to the phase of the laser was extensively investigated [22].

Importantly, recent theoretical and experimental papers have highlighted that topological excitations 
(vortex-antivortex pairs and bound dark soliton pairs) can be stabilized in driven dissipative condensates when working within the optical bistability regime under quasiresonant pumping configuration. The formation of complex phase structures, when working in the bistable regime, demonstrates the possibility to overcome the phase fixing problem typical of strong resonant pumping [23-25]. Within the bistable hysteresis cycle, two solutions are available for the system, respectively at high and low polariton density for the same pumping density. At high density above the bistability cycle, the phase of the quantum fluid wave function is fixed by the one of the resonant laser. Recent theoretical studies [26] also investigated frequency and phase synchronization mechanism occurring for stable states of an incoherently pumped polariton condensate, showing similar results.

In this paper, we report the observation of new quantum hydrodynamic effects revealed by using the optical bistability of exciton-polaritons in microcavities under quasiresonant pumping. We experimentally demonstrate the hydrodynamic generation of a parallel dark soliton pair in the wake of a cavity structural defect. This bound soliton pair propagates along the polariton flow as long as the laser pumping sustains it, thus removing the constraints imposed by the polariton lifetime in previous experimental configurations.

The device under investigation is a GaAs/AlGaAs microcavity with 21 (front) and 24 (back) layers of DBR and $\mathrm{In}_{0.04} \mathrm{Ga}_{0.96} \mathrm{As}$ quantum wells at each of the three antinodes of the confined electromagnetic field [27]. A small wedge is inserted between the Bragg mirrors during the fabrication process, allowing us to precisely select the cavity-exciton detuning. All experiments are performed at $10 \mathrm{~K}$. The exciton energy is $1.485 \mathrm{eV}$, the cavity exciton-photon detuning is negative $(-1.4 \mathrm{meV})$, and the half Rabi splitting is $2.55 \mathrm{meV}$ [see Fig. 1(a)]. The polariton mass, extracted from the dispersion, is $7 \times 10^{-5}$ free electron mass. The polariton lifetime is 14 ps. A continuous-wave (cw) single-mode laser excites the polaritons in the quasiresonant regime. The experiments are performed in transmission configuration; i.e., the excitation and the detection are on opposite sides of the sample so that the signal is not blinded by the resonant pump laser. In order to work within the optical bistability regime, the laser frequency is slightly blue detuned $(0.16 \pm 0.02 \mathrm{meV})$ with respect to the lower polariton dispersion branch, guaranteeing the generation of a hysteresis loop [28]; see Fig. 1(b).

The present configuration implements the effective pump and support scheme proposed in Ref. [23] for sustaining the propagation of topological defects in dissipative polariton quantum fluids. In such scheme, a strong localized pump drives the system to the upper bistability branch. This pump is surrounded by a weak support beam with the intensity falling within a bistability loop. This condition permits us to overcome the polariton decay while allowing the generation of collective excitations with a peculiar phase texture $[23,29,30]$. In our experiment, the pump beam center is positioned slightly upstream of an obstacle (cavity structural defect) and its power is chosen to efficiently drive the system to the upper branch of the bistability loop [Fig. 2(a)]. Concerning the support beam, cylindrical lenses are used to shape it and to make it elliptic $(100 \times 400 \mu \mathrm{m}$ FWHM $)$ with the flow direction along the major ellipse axis. The pump beam is near the center
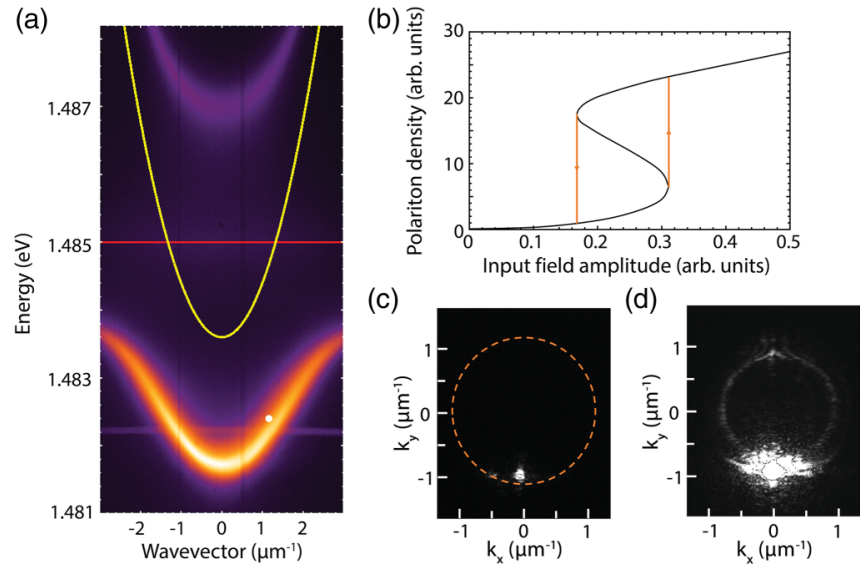

FIG. 1. Cavity dispersion and bistability. (a) Energy dispersion of the exciton-polariton emission when the microcavity is pumped with a nonresonant $\mathrm{cw}$ laser at $1.54 \mathrm{eV}$. The polariton density is in logarithmic scale. The bare photon and exciton dispersions are in yellow and red curves, respectively. The white dot corresponds to the position of the pump, slightly blue detuned from the lower polariton branch. (b) Theoretical calculations of the bistability loop according to the experimental parameters given in the text. (c) Output intensity distribution in the momentum space when the system is in the lower branch of the bistability loop and with an excitation at $k=1.2 \mu \mathrm{m}^{-1}$ and energy $1482.4 \mathrm{meV}$. The orange dashed circle depicts the Rayleigh scattering ring, which is visible for longer integration time (higher contrast). (d) Output intensity distribution in the momentum space when the system jumps to the upper branch of the bistability loop. The scattering ring is now visible and it has a smaller radius compared to the one in panel (c).

of the support beam. The pump and support beams have the same in-plane wave vectors. As far as both these beams are obtained by splitting the same initial laser beam (elsewhere in the optical path of the setup), they remain mutually coherent.

The polariton group velocity is finely tuned by choosing the angle of incidence $\theta$ of the pump. Indeed, the in-plane wave vector $\left(k=k_{0} \sin \theta \approx 1.2 \mu^{-1}\right.$ [see Fig. 1(c) and $1(\mathrm{~d})]$, where $k_{0}$ is the wave vector of the pumping laser) and determines the group velocity according to the polariton dispersion relation $\left(v_{\text {flow }}=1.52 \mu \mathrm{m} / \mathrm{ps}\right)$ reported in Fig. 1(a). In such quantum fluid, the sound speed reads $c_{s}=\sqrt{g|\psi|^{2} / m}$, where $g$ is the polariton-polariton interaction constant, $m$ is the polariton mass, and $|\psi|^{2}$ is the polariton density. Experimentally, the speed of sound was determined by measuring the energy renormalization (blue shift) when the system jumps in the upper bistability branch. In our experimental configuration, this increase in energy corresponds with a good approximation (few percents) to the product $g|\psi|^{2}$. In our experimental conditions $c_{s} \approx 0.6 \mu \mathrm{m} / \mathrm{ps}$, which means that the obtained flow is supersonic. Previous observations of dark solitons in purely dissipative polariton systems, i.e., without the support beam, were achieved for flow velocities slightly supersonic and even subsonic [31]. In the configuration studied in this work, the dissipation is compensated by the presence of the support beam, which ensures a nearly constant polariton density along the device. Experimentally, we observe stable dark solitons only for deeply supersonic flows. Indeed, when working in the subsonic regime, the solitons 
(a)

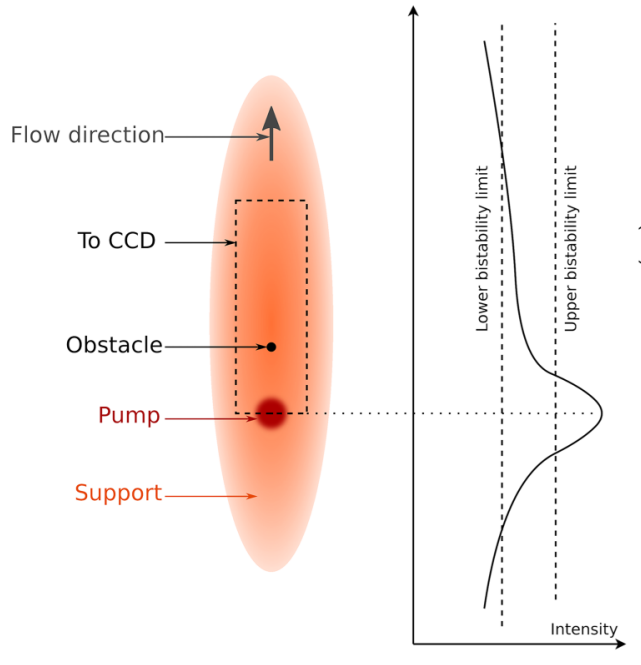

(b)

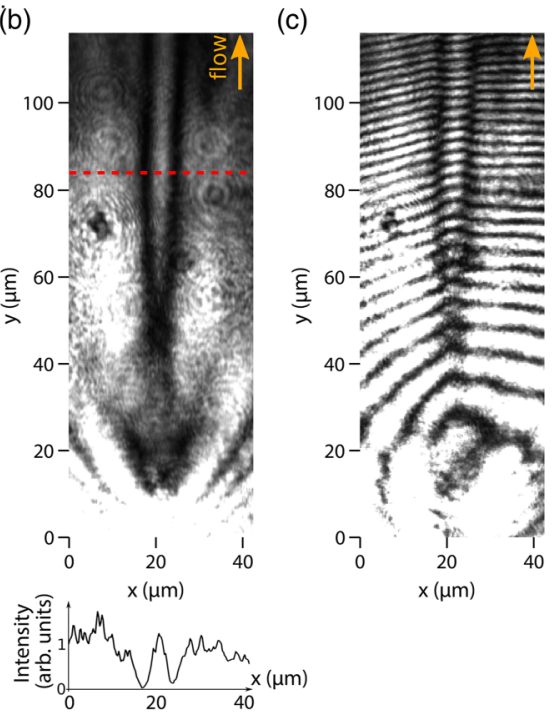

(d)

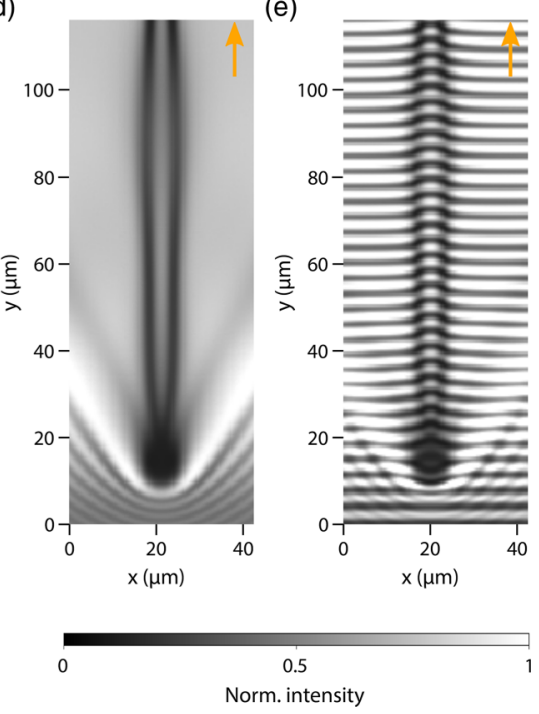

FIG. 2. (a) Experiment scheme with pump and support beams. The curve sketches the laser intensity along the propagation axis. (b) Intensity map of the polariton fluid flowing across a structural defect. The turbulences generated in the wake of the defect evolve into two parallel dark solitons. The red dotted line indicates the position of the transverse section presented in the inset. (c) Interference pattern associated to panel (b), showing a phase jump close to $\pi$ across the solitons. (d) Theoretical simulations (using the driven dissipative Gross-Pitaevskii equation) of the intensity map for parameters corresponding to those used in the experiment. (e) Theoretical interference pattern corresponding to panel (d).

generation is inhibited and replaced by the formation of a vortex stream in the wake of the defect [29].

The healing length is $\xi=\hbar / \sqrt{2 m g|\psi|^{2}} \approx 1.8 \mu \mathrm{m}$.

In the geometry described above, with the support beam close to the lower limit of the upper bistability branch, we observe the hydrodynamic formation of a soliton pair in the shadow of the defect [Fig. 2(b)]. The intensity of the pump does not strongly affect the results because its only role is to locally maintain the system to the upper bistability branch. In Fig. 2(b), the polariton density decreases slightly in the zone pumped by the support beam, and then the effect on the sound velocity is quite small. During the first $40 \mu \mathrm{m}$ of their propagation, the solitons reach an equilibrium separation distance (about $8 \mu \mathrm{m}$ ) and then they continue a parallel propagation for about the next $45 \mu \mathrm{m}$. The inset of Fig. 2(b) represents a transverse section across the solitons corresponding to the red line in the main figure. It clearly shows that the solitons are fully dark with a density depth reaching zero at the center of the soliton. From the interference map obtained by homodyne detection [Fig. 2(c)], one also sees that the phase jump across each soliton is very close to $\pi$ when the two dark solitons align to each other, which means their transverse velocity is close to zero. The two parallel solitons are fully dark and their width is almost constant since it is related to the healing length, which is, in turn, almost constant in the whole area locked to the upper bistability branch.

To reproduce the soliton behavior observed in the experiments, we have performed numerical simulations based on the coupled equations for the excitons $\left(\psi_{X}\right)$ and cavity photon $\left(\psi_{P h}\right)$ fields:

$$
\begin{aligned}
i \hbar \frac{\partial \psi_{\mathrm{Ph}}(\mathbf{r}, t)}{\partial t} & =\left[-\frac{\hbar^{2} \nabla^{2}}{2 m}+V(\mathbf{r})-i \Gamma_{\mathrm{Ph}}\right] \psi_{\mathrm{Ph}}(\mathbf{r}, t)+V \psi_{\mathrm{X}}(\mathbf{r}, t)+[S(\mathbf{r})+P(\mathbf{r})] e^{-i \omega_{0} t}, \\
i \hbar \frac{\partial \psi_{\mathrm{X}}(\mathbf{r}, t)}{\partial t} & =\left[V(\mathbf{r})+g_{X}\left|\psi_{\mathrm{X}}(\mathbf{r}, t)\right|^{2}-i \Gamma_{\mathrm{X}}-\Delta_{\mathrm{X}}\right] \psi_{\mathrm{X}}(\mathbf{r}, t)+V \psi_{\mathrm{Ph}}(\mathbf{r}, t) .
\end{aligned}
$$

The parameters (cavity photon mass $m$, laser energy $\omega_{0}$, photon lifetimes $\Gamma_{\mathrm{Ph}}$, half-Rabi splitting $V$, and cavity-exciton detuning $\Delta_{X}$ ) were taken to be the same as in the experiment. The value used in the simulations is $g_{X}=0.01 \mathrm{meV} / \mu \mathrm{m}^{2}$, as it has been determined in previous experiments using the same sample [32]. The exciton lifetime $\Gamma_{\mathrm{X}}$ was taken to be $150 \mathrm{ps}$. The obstacle was modeled as a $10-\mathrm{meV}$ potential barrier $V(\mathbf{r})$ with a Gaussian shape $10 \mu \mathrm{m}$ wide. The relative positions of the obstacle and pump beam also reproduce the experimental configuration. $P(\mathbf{r})$ and $S(\mathbf{r})$ describe the spatial profiles of the pump and support beams, respectively, and their magnitudes are adjustable parameters. The output images reflect the spatial profile of the photon component density $\left|\psi_{\mathrm{Ph}}(\mathbf{r}, t)\right|^{2}$ and the interference pattern $\left|\psi_{\mathrm{Ph}}(\mathbf{r}, t)+A_{0} e^{-i \mathbf{k}_{0} \mathbf{r}}\right|^{2}$. The results of the simulations [Figs. 2(d) and 2(e)] are in excellent agreement with the corresponding experimental images.

The pump creates a flow which is large enough to switch the area below the support on the upper bistability branch. This dynamically occurs during the establishment of the steady state, through the propagation of domain walls 
separating the regions being on the upper and lower branch of bistability respectively [24]. The perturbations in the wake of the potential barrier made by the defect evolves into stable soliton pairs. The area in between the solitons has weak laser absorption and most of the particles come from the surrounding high density regions. Therefore, its phase is not fixed by the forcing field and the intensity and phase distributions result from the collective excitation sustained by the system. A stationary soliton pattern must contain an even number of dark solitons because the phase jump across a dark soliton is $\pi$ and because the total phase change between two highdensity regions having the same phase has to be a multiple of $2 \pi$. The stability of the soliton molecule can therefore be understood in terms of a balance between the well-known repulsive interaction between dark solitons [33], which tends to push away the solitons, and the pressure exerted by the external particles which tends to propagate toward the center in order to recover a uniform phase in the whole are below the support. Numerical simulations performed for different parameters have evidenced the important role of the polariton dissipation rate on the solitons' equilibrium distance. Quite surprisingly, the solitons' distance is weakly dependent on the Mach number, and the solitons align closer to each other for increasing dissipation rates. This suggests that the equilibrium between the domain wall pressure and the soliton repulsion is governed by the refill of the area in between the solitons which is mainly due to the polariton tunneling across the soliton: The refilling is more efficient for low polariton dissipation rates and therefore the equilibrium distance decreases for increasing polariton dissipation rates [34].

A detailed experimental analysis of the equilibrium distance with respect to any of these parameters is extremely hard to perform, since their tuning rapidly leads to the disappearance of the soliton pair due to the phase locking of the whole excited area or, when lowering the support intensity, to the collapse of the system to the lower bistability branch.

Moreover, the propagation distance of the soliton pair is limited by both the sample wedge and the finite size of the support laser. At some distance, due to the Gaussian shape of the support beam, the flow falls into the linear regime, with low polariton density and the soliton pair cannot be sustained anymore.

In order to compare the bound soliton pair to the solitons generated in a purely dissipative regime, we have performed an experiment similar to the one in Ref. [15], that is, removing the support beam (Fig. 3). In order to avoid the phase fixing of the polaritons to the laser phase, which inhibits the solitons generation, the pump beam is exciting the sample at a distance of about $20 \mu \mathrm{m}$ upstream of the defect position, and thus it does not overlap with the structural defect. The solitons, spontaneously generated along the flow of the fluid, are oblique. In this case, unlike the previous configuration, the expansion of the inner region between the solitons is not inhibited by the presence of the out-of-phase support beam. Therefore, the separation distance between the solitons increases along the flow, due to their repulsive interaction. Moreover, as is visible in the inset of Fig. 3, the oblique solitons are gray and they have a large width (10 $\mu \mathrm{m}$ FWHM) compared to the width observed for parallel solitons obtained in the driven-dissipative regime with the support beam and within the bistability loop
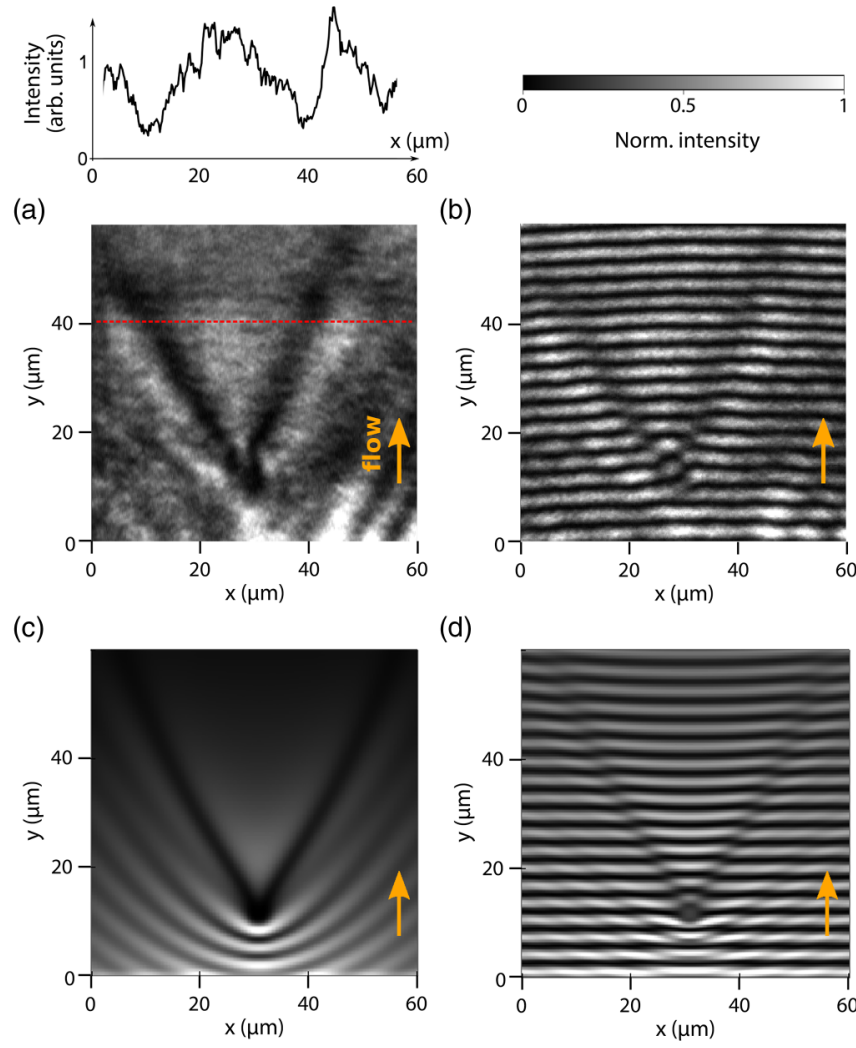

(d)

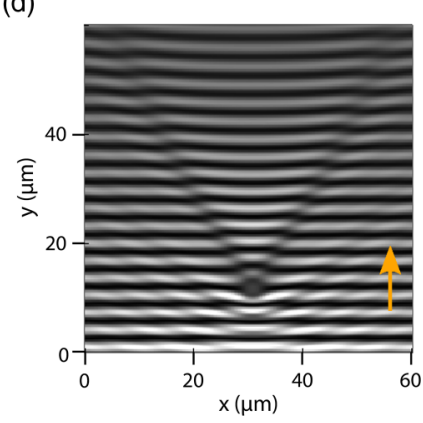

FIG. 3. Oblique solitons. (a) Intensity map of the polariton flowing across a structural defect and excited using a semicircular shaped pump upstream to the defect. The generated solitons in the wake of the defect are gray, as shown in the inset on top corresponding to the transverse section indicated by the red dashed line, and they propagate with increasing separation distance. (b) Interference pattern associated to panel (a). The phase jump is decreasing along the propagation as the solitons vanish. [(c), (d)] Panels are the theoretical simulations of the intensity and interference maps, respectively.

(4.3 $\mu \mathrm{m}$ FWHM). The theoretical simulations perfectly reproduce the experimental results [Fig. 3(c) and 3(d)]. Without the support beam, the polariton density decreases: As a result, the sound velocity and the fluid velocity change along the propagation. Nevertheless, the solitons still present an almost rectilinear shape, because the decrease in the speed of sound is compensated by the acceleration of the solitons when they become less deep. Furthermore, the oblique solitons vanish soon because of the decrease of the mean polariton density without the support. Indeed, the fluid propagation length is $19.6 \mu \mathrm{m}\left(v_{\text {flow }}=1.4 \mu \mathrm{m} / \mathrm{ps}\right)$ and it limits the soliton propagation distance at about $30 \mu \mathrm{m}$.

Figure 4 shows the separation distance of the two kinds of solitons, oblique and parallel, along their propagation (orange and black dashed lines respectively). The black solid line in Fig. 4 is the fit of the experimental soliton distance with the function $2 d_{0}\left(1-\exp \left(-x / d_{l}\right)\right)$. The initial transient region, where the solitons are oblique, has a characteristic length $d_{l}=$ $14 \mu \mathrm{m}$, and then the solitons reach the equilibrium distance, with an asymptotic separation of $2 d_{0}=8 \mu \mathrm{m}$. On the other hand, the separation distance of the oblique solitons from Fig. 3 keeps increasing with the propagation. 


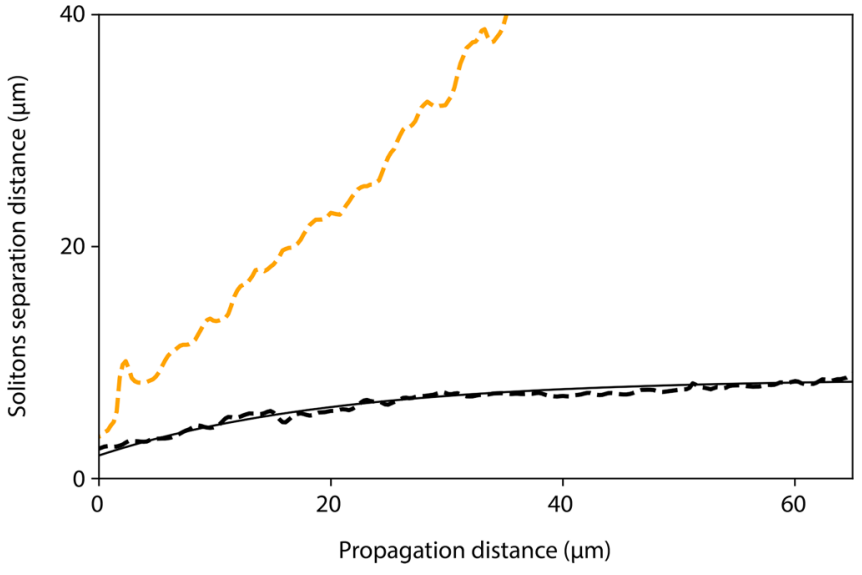

FIG. 4. Dark solitons separation distance for parallel (black dashed line) and oblique (orange dashed line) solitons along their in-plane propagation. The black solid line is the fitting of the parallel solitons experimental data (see main text for further details).

Our experimental results demonstrate that collective excitations with rich phase behavior can be generated in a polariton superfluid within the bistable regime and detected on large length scales in $\mathrm{cw}$ experiments. The presence of the support beam allows the formation of a stable bound soliton pair. The key advantage of this experimental configuration is the possibility to decouple the collective excitations lifetime from the polariton one. Indeed, while the solitons propagation length without support is limited by the polariton lifetime, in the bistable regime the collective excitations are sustained by a constant polariton density along their propagation, and therefore they can propagate as long as the system is on the bistability regime. In our experiment, the bound solitons propagate on a much longer distance (about $80 \mu \mathrm{m}$ ) than the oblique solitons without a support beam (about $30 \mu \mathrm{m}$ ). These results open perspectives for probing the hydrodynamic evolution of collective excitations, quantum turbulence, and mesoscopic-scale physics in out-of-equilibrium condensates.

We acknowledge the support of the ANR projects "Quantum Fluids of Light" (ANR-16-CE30-0021) and C-FLigHT (ANR-16-ACHN-0027) and of the ANR program "Investissements d'Avenir" through the IDEX-ISITE initiative 16-IDEX-0001 (CAP 20-25). This work has received funding from the European Unions Horizon 2020 research and innovation programme under Grant Agreement No. 820392 (PhoQuS). S.V.K. acknowledges the support from the Ministry of Education and Science of Russian Federation (No. 0791-2020-0006). G.L. would like to thank Iacopo Carusotto for useful discussions.
[1] K. B. Davis, M.-O. Mewes, M. R. Andrews, N. J. van Druten, D. S. Durfee, D. M. Kurn, and W. Ketterle, Bose-Einstein Condensation in a Gas of Sodium Atoms, Phys. Rev. Lett. 75, 3969 (1995).

[2] J. Kasprzak, M. Richard, S. Kundermann, A. Baas, P. Jeambrun, J. Keeling, F. Marchetti, M. Szymańska, R. Andre, J. Staehli et al., Bose-Einstein condensation of exciton polaritons, Nature (London) 443, 409 (2006).

[3] J. R. Anglin and W. Ketterle, Bose-Einstein condensation of atomic gases, Nature (London) 416, 211 (2002).

[4] T. Byrnes, N. Y. Kim, and Y. Yamamoto, Exciton-polariton condensates, Nat. Phys. 10, 803 (2014).

[5] M. R. Matthews, B. P. Anderson, P. C. Haljan, D. S. Hall, C. E. Wieman, and E. A. Cornell, Vortices in a Bose-Einstein Condensate, Phys. Rev. Lett. 83, 2498 (1999).

[6] S. Burger, K. Bongs, S. Dettmer, W. Ertmer, K. Sengstock, A. Sanpera, G. V. Shlyapnikov, and M. Lewenstein, Dark Solitons in Bose-Einstein Condensates, Phys. Rev. Lett. 83, 5198 (1999).

[7] K. G. Lagoudakis, M. Wouters, M. Richard, A. Baas, I. Carusotto, R. André, L. S. Dang, and B. Deveaud-Plédran, Quantized vortices in an exciton-polariton condensate, Nat. Phys. 4, 706 (2008).

[8] G. Roumpos, M. D. Fraser, A. Löffler, S. Höfling, A. Forchel, and Y. Yamamoto, Single vortex-antivortex pair in an excitonpolariton condensate, Nat. Phys. 7, 129 (2011).

[9] G. Tosi, G. Christmann, N. Berloff, P. Tsotsis, T. Gao, Z. Hatzopoulos, P. Savvidis, and J. Baumberg, Geometrically locked vortex lattices in semiconductor quantum fluids, Nat. Commun. 3, 1243 (2012).
[10] D. Sanvitto, S. Pigeon, A. Amo, D. Ballarini, M. De Giorgi, I. Carusotto, R. Hivet, F. Pisanello, V. Sala, P. Guimaraes et al., All-optical control of the quantum flow of a polariton condensate, Nat. Photon. 5, 610 (2011).

[11] G. Lerario, A. Fieramosca, F. Barachati, D. Ballarini, K. Daskalakis, L. Dominici, M. De Giorgi, S. A. Maier, G. Gigli, and D. Sanvitto, Room-temperature superfluidity in a polariton condensate, Nat. Phys. 13, 837 (2017).

[12] G. Gauthier, M. T. Reeves, X. Yu, A. S. Bradley, M. A. Baker, T. A. Bell, H. Rubinsztein-Dunlop, M. J. Davis, and T. W. Neely, Giant vortex clusters in a two-dimensional quantum fluid, Science 364, 1264 (2019).

[13] S. P. Johnstone, A. J. Groszek, P. T. Starkey, C. J. Billington, T. P. Simula, and K. Helmerson, Evolution of large-scale flow from turbulence in a two-dimensional superfluid, Science 364, 1267 (2019).

[14] G. Grosso, G. Nardin, F. Morier-Genoud, Y. Léger, and B. Deveaud-Plédran, Soliton Instabilities and Vortex Street Formation in a Polariton Quantum Fluid, Phys. Rev. Lett. 107, 245301 (2011).

[15] A. Amo, S. Pigeon, D. Sanvitto, V. Sala, R. Hivet, I. Carusotto, F. Pisanello, G. Leménager, R. Houdré, E. Giacobino et al., Polariton superfluids reveal quantum hydrodynamic solitons, Science 332, 1167 (2011).

[16] E. Wertz, A. Amo, D. D. Solnyshkov, L. Ferrier, T. C. H. Liew, D. Sanvitto, P. Senellart, I. Sagnes, A. Lemaître, A. V. Kavokin, G. Malpuech, and J. Bloch, Propagation and Amplification Dynamics of 1d Polariton Condensates, Phys. Rev. Lett. 109, 216404 (2012). 
[17] A. Kavokin, J. J. Baumberg, G. Malpuech, and F. P. Laussy, Microcavities (Oxford University Press, Oxford, UK, 2011).

[18] I. Carusotto and C. Ciuti, Quantum fluids of light, Rev. Mod. Phys. 85, 299 (2013).

[19] E. A. Kuznetsov and S. K. Turitsyn, Instability and collapse of solitons in media with a defocusing nonlinearity, Zh. Eksp. Teor. Fiz. 94, 119 (1988).

[20] J. Brand and W. P. Reinhardt, Solitonic vortices and the fundamental modes of the snake instability: Possibility of observation in the gaseous Bose-Einstein condensate, Phys. Rev. A 65 , 043612 (2002).

[21] A. M. Kamchatnov and L. P. Pitaevskii, Stabilization of Solitons Generated by a Supersonic Flow of Bose-Einstein Condensate Past an Obstacle, Phys. Rev. Lett. 100, 160402 (2008).

[22] V. Goblot, H. S. Nguyen, I. Carusotto, E. Galopin, A. Lemaître, I. Sagnes, A. Amo, and J. Bloch, Phase-Controlled Bistability of a Dark Soliton Train in a Polariton Fluid, Phys. Rev. Lett. 117, 217401 (2016).

[23] S. Pigeon and A. Bramati, Sustained propagation and control of topological excitations in polariton superfluid, New J. Phys. 19, 095004 (2017).

[24] S. V. Koniakhin, O. Bleu, D. D. Stupin, S. Pigeon, A. Maitre, F. Claude, G. Lerario, Q. Glorieux, A. Bramati, D. Solnyshkov et al., Stationary Quantum Vortex Street in a Driven-Dissipative Quantum Fluid of Light, Phys. Rev. Lett. 123, 215301 (2019).

[25] P. Parra-Rivas, E. Knobloch, D. Gomila, and L. Gelens, Dark solitons in the Lugiato-Lefever equation with normal dispersion, Phys. Rev. A 93, 063839 (2016).
[26] I. Chestnov, A. Kavokin, and A. Yulin, The optical control of phase locking of polariton condensates, New J. Phys. 21, 113009 (2019).

[27] R. Houdré, C. Weisbuch, R. P. Stanley, U. Oesterle, and M. Ilegems, Coherence effects in light scattering of twodimensional photonic disordered systems: Elastic scattering of cavity polaritons, Phys. Rev. B 61, R13333(R) (2000).

[28] A. Baas, J. P. Karr, H. Eleuch, and E. Giacobino, Optical bistability in semiconductor microcavities, Phys. Rev. A 69, 023809 (2004).

[29] G. Lerario, A. Maître, R. Boddeda, Q. Glorieux, E. Giacobino, S. Pigeon, and A. Bramati, Vortex-stream generation and enhanced propagation in a polariton superfluid, Phys. Rev. Research 2, 023049 (2020).

[30] A. V. Yulin, O. A. Egorov, F. Lederer, and D. V. Skryabin, Dark polariton solitons in semiconductor microcavities, Phys. Rev. A 78, 061801(R) (2008).

[31] A. A. M. Kamchatnov and S. V. Korneev, Oblique solitons generated by the flow of a polariton condensate past an obstacle, J. Exp. Theor. Phys. 1115, 579 (2012).

[32] A. Amo, J. Lefrère, S. Pigeon, C. Adrados, C. Ciuti, I. Carusotto, R. Houdré, E. Giacobino, and A. Bramati, Superfluidity of polaritons in semiconductor microcavities, Nat. Phys. 5, 805 (2009).

[33] Y. S. Kivshar and W. Królikowski, Lagrangian approach for dark solitons, Opt. Commun. 114, 353 (1995).

[34] A. Maître, G. Lerario, A. Medeiros, F. Claude, Q. Glorieux, E. Giacobino, S. Pigeon, and A. Bramati, Dark-Soliton Molecules in an Exciton-Polariton Superfluid, Phys. Rev. X 10, 041028 (2020). 